\title{
Gamification in Unternehmen
}

\section{Beeinflussung der Unternehmenskultur durch spieletypische Elemente}

\author{
Thomas Ellenberger, Deane Harder und Marie Brechbühler Pešková
}

\begin{abstract}
Zusammenfassung
Eine ,gute“ Unternehmenskultur ist ein wichtiger Erfolgsfaktor für Unternehmen. Im Rahmen der digitalen Transformation wird zunehmend Gamification zur Kulturbeeinflussung eingesetzt. Dabei werden spieletypische Elemente genutzt, um für Mitarbeitende für bestimmte Handlungen Anreize zu schaffen. Im Rahmen einer Fallstudie wurden digital umgesetzte Gamification-Maßnahmen eines Unternehmens bezüglich ihrer Wahrnehmung und Beeinflussung der Arbeitsmotivation analysiert. Die Ergebnisse zeigen, dass Gamification kulturwirksam zur Unternehmensführung genutzt werden kann. Die digitale Umsetzung diente als einheitlicher Impulsgeber und sorgte für Transparenz als Basis für Vertrauen. So konnte die angestrebte „Kultur auf Augenhöhe“ entwickelt werden, geprägt durch Respekt, Toleranz und Mitbestimmung. Der Erfolg der Gamification-Maßnahmen lässt sich vor allem durch die positive Wirkung auf die intrinsische Motivation sowie das gestärkte Zusammengehörigkeitsgefühl der Mitarbeitenden erklären.
\end{abstract}

T. Ellenberger $(\bowtie)$

Bern, Schweiz

E-Mail: thomas.ellenberger@gmx.ch

D. Harder

Berner Fachhochschule Wirtschaft, Bern, Schweiz

E-Mail: deane.harder@bfh.ch

M. Brechbühler Pešková

Institut Unternehmensentwicklung, Berner Fachhochschule Wirtschaft, Bern, Schweiz

E-Mail: marie.brechbuehler@bfh.ch

J. Schellinger et al. (Hrsg.), Digitale Transformation und Unternehmensführung,

https://doi.org/10.1007/978-3-658-26960-9_4 


\subsection{Einleitung}

„In every job that must be done, there is an element of fun.

You find the fun, and - SNAP - the job's a game!“"

Mary Poppins

Im Verständnis vieler Experten ist der Mensch ein spielendes Wesen, ein Homo ludens. Rund 2,2 Mrd. Gamer verbringen weltweit mehr als $3 \mathrm{Mrd}$. h pro Woche mit Videospielen (McDonald 2017; McGonigal 2012). Das ergibt etwa 156 Mrd. h pro Jahr. Videospiele sind längst keine Randerscheinung mehr, sie wurden während den letzten Jahren immer mehr zu einem Massenphänomen. Dabei werden die allermeisten Menschen nicht fürs Spielen bezahlt, sondern investieren ihre Zeit auf freiwilliger Basis. Mit dem enormen Erfolg von Videospielen kam in den vergangenen Jahren der Trend auf, spieletypische Elemente auch in spielefremde Kontexte zu verwenden. Dieses Vorgehen wird als Gamification bezeichnet. Tätigkeiten, Arbeitsabläufe oder ganze Prozesse können mithilfe von spielerischen Attributen und Prinzipien für die Anwender interessanter gestaltet werden.

Demografischer Treiber dieser Entwicklung sind vor allem die Generationen X, Y und Z. Also jene Menschen, die seit kurz vor der Jahrtausendwende geboren wurden und nun immer mehr in die Arbeitswelt drängen. Viele von ihnen sind sich durch ihre Freizeitaktivitäten eine ständige Stimulation der Sinne gewohnt und mögen es, mit Gamification-Elementen angesprochen zu werden (Ryan et al. 2013). Sie verlangen nach Spielerlebnissen in einer Art in der Freizeit, aber auch zunehmend bei der Arbeit, die ältere Generationen oft wenig verstehen (McGonigal 2012). Sie wollen eine erfüllende, für sie sinnvolle Tätigkeit ausüben, in der sie ihr Können einbringen und die Möglichkeit zur Selbstverwirklichung haben (Wikipedia 2018). Gamification verspricht genau diese Anforderungen erfüllen zu können. Mit entsprechend gestalteten Arbeitssituationen soll die Arbeitsmotivation der Mitarbeitenden gezielt und positiv angesprochen werden.

\subsubsection{Zielsetzungen der Studie}

Ziel dieser Studie war das Analysieren und Dokumentieren von digital umgesetzten Gamification-Maßnahmen zur Kulturbeeinflussung in Unternehmen. Dafür wurden die Maßnahmen der Firma Acme Apps $A G^{1}$ mit dem Octalysis-Modell zur Gamification untersucht und aufbereitet. Zudem sollte herausgefunden werden, wie die Gamification-Maßnahmen von Mitarbeitenden wahrgenommen werden und welche Wirkung diese auf die Arbeitsmotivation von Einzelnen und die Unternehmenskultur insgesamt haben.

\footnotetext{
${ }^{1}$ Name geändert.
} 


\subsubsection{Relevanz des Themas}

Bereits im Jahr 2013 zählte das Wirtschaftsmagazin Forbes Gamification zu einem der wichtigsten disruptiven Trends. Damals, auf dem Höhepunkt des sogenannten Gartner Hype Cycles, wurde jedoch auch vorhergesagt, dass bereits im Jahr 2014 etwa $80 \%$ der bestehenden Gamification-Maßnahmen aufgrund von fehlerhaftem Design fehlschlagen würden (Burke 2013). Im Jahr 2015 erschien der Begriff Gamification zum letzten Mal auf dem Gartner Hype Cycle. Gemäß diesem war die Zeit der übertriebenen Erwartungen an die Gamification vorbei und der Trend befand sich in der Phase des „Tals der Enttäuschung“. Es wurde vorausgesagt, dass der Trend in 2 bis 5 Jahren zum Mainstream gehören und das „Plateau der steigenden Produktivität“ erreichen würde (Levy 2015). Ein Jahr später, im August 2016, prognostizierte Forbes, Gamification würde bereits ab dem Jahr 2017 zu einer der zentralen Business-Strategien zählen (Newman 2016). In den Jahren des Hypes litt die Reputation von Gamification als unternehmerische Maßnahme. Wie durch Burke (2013) vorhergesagt, waren viele der bis dahin implementierten Maßnahmen unausgereift und deren Resultate konnten den hohen Erwartungen nicht gerecht werden.

Gemäß Gamification-Experten Yu-kai Chou (2016) lag dies an der Überbetonung bestimmter Gamification-Elemente. Solche fehldesignten Maßnahmen zeichnen sich dadurch aus, dass sie vor allem auf Punkte, Abzeichen und Ranglisten fokussieren (englisch Points, Badges and Leaderboards oder kurz PBL). Zur besseren und einfacheren Gestaltung von Gamification-Maßnahmen in der Praxis entwickelte Chou das sogenannte Octalysis-Modell (Chou 2018). Es eignet sich aber auch, um bereits bestehende Maßnahmen zu analysieren und bildet die methodische Grundlage der vorliegenden Studie.

\subsection{Unternehmenskultur, Gamification und Motivation}

Zum Verständnis der Studie werden nachfolgend einige der zentralen Begriffe theoretisch aufgearbeitet und definiert. Zuerst werden die Theorien zur Unternehmenskultur und zur Motivation vorgestellt. Ziel des Einbezugs dieser Theorien ist in erster Linie die Erarbeitung einer einheitlichen Terminologie. Daran anschließend wird Gamification erläutert und ein Überblick darüber gegeben, was das Thema alles beinhaltet und wie dieses in der Praxis angewendet werden kann. Ergänzt wird die Theorie durch die Vorstellung des Octalysis-Modells, das zur Analyse der Maßnahmen zur Kulturbeeinflussung verwendet wurde. 


\subsubsection{Definition Unternehmenskultur}

Einer der ersten und bis heute mit am weitesten verbreiteten Definition zur Unternehmenskultur stammt von Edgar H. Schein (1984). Er definiert Kultur als: „ein Muster gemeinsamer Grundprämissen, das die Gruppe bei der Bewältigung ihrer Probleme mit externen Anpassungen und interner Integration erlernt hat, das sich bewährt hat und das somit als bindend gilt; und das daher an neue Mitglieder als rational und emotional korrekter Ansatz für den Umgang mit diesen Problemen weitergegeben wird. " Herget und Strobl (2018) betonen dagegen eher normative Aspekte: „Kultur stellt die zentralen Spiel- und Kommunikationsregeln auf, die das faktische Leben innerhalb der Organisation entscheidend prägen. Sie legt auf eine informelle Art und Weise fest ,was man hier macht und was nicht', was als ,gut' belohnt wird und was als ,schlecht" sanktioniert wird.“ Eine ähnliche Definition bietet Sackmann (2002): „Unternehmenskultur sind die gemeinsamen grundlegenden Überzeugungen in einer Gruppe, die diese charakterisieren. Diese Überzeugungen beeinflussen Wahrnehmung, Denken, Handeln und Fühlen der Gruppenmitglieder. Sie können sich auch in den Artefakten manifestieren. Die Überzeugungen werden nicht mehr bewusst gehalten, sie sind aus der Erfahrung der Gruppe entstanden und haben sich durch die Erfahrung der Gruppe weiterentwickelt, d. h. sie sind gelernt und werden an neue Gruppenmitglieder weitergegeben“ (Sackmann 2002). Zentral bei diesen Definitionen sind also die impliziten Spielregeln und dass Kultur emergent und evolutionär ist, sie also von sich aus erwächst und nicht geplant oder verordnet werden kann.

\subsubsection{Beeinflussung der Unternehmenskultur}

Studien haben gezeigt, dass eine starke Unternehmenskultur mit dem Erfolg eines Unternehmens korreliert (Kotter und Heskett 1992). So wurde herausgefunden, dass mit einer „guten“ Unternehmenskultur sowohl der Umsatz, der Nettogewinn, der Aktienkurs sowie der Bestand an Mitarbeitenden signifikant stärker wuchsen (Jost 2003). Die Beeinflussung der Unternehmenskultur in eine positive Richtung ist also stark im Interesse des Unternehmens.

Eine Unternehmenskultur kann nicht direkt verändert werden. Um eine Unternehmenskultur verändern zu können, ist es zunächst wichtig, dass die Führungskräfte einen Veränderungsbedarf erkennen und gewillt sind, eine Veränderung herbeizuführen. In einem zweiten Schritt sollten die Mitarbeitenden für den Prozess gewonnen und ihnen die Notwendigkeit zur Veränderung plausibel und glaubwürdig vermittelt werden. Für die Erarbeitung der tatsächlichen Maßnahmen sollten möglichst viele Betroffene miteinbezogen und die Maßnahmen gemeinsam erarbeitet werden. Mitarbeitende sollten dabei jederzeit das Gefühl haben, vom Management unterstützt zu werden. Ansonsten kann der ganze Prozess nach einer anfänglichen Euphorie schnell im Sand verlaufen. Anschließend ist eine ständige Überprüfung und Weiterentwicklung der Kultur und der Maßnah- 
men sinnvoll (Zelesniack und Grolman 2018). Auch wenn der prinzipielle Prozess relativ einfach ist, sollte stets bedacht werden, dass Kulturveränderungen nicht so zielgerichtet bewirkt werden kann wie die Umsetzung eines Projektplans. Vielmehr bedarf es einen kontinuierlichen Abgleich zwischen gewünschter und tatsächlicher Wirkung von Maßnahmen und entsprechenden Anpassungen im weiteren Vorgehen.

\subsection{Motivation}

Motivation ist ein oft verwendeter Ausdruck, wenn es darum geht, etwas Bestimmtes anzupacken oder sein zu lassen. Motivation ist der ,Zustand einer Person, der sie dazu veranlasst, eine bestimmte Handlungsalternative auszuwählen, um ein bestimmtes Ergebnis zu erreichen und der dafür sorgt, dass diese Person ihr Verhalten hinsichtlich Richtung und Intensität beibehält" (Maier und Kirchgeorg 2018). Etwas anschaulicher ist Motivation die Triebkraft, die uns dazu bringt, bei mehreren Optionen die für uns ansprechendste auszuwählen und uns dazu bringt, eine Aktivität weiter zu verfolgen. Unsere Motivation ist dabei immer abhängig von einem aktuellen Bedürfnis. Zudem bestimmt sie, mit welcher Intensität und welcher Ausdauer wir uns in welche Richtung bewegen (De Luca 2017). Die gleiche Aufgabe kann dabei verschiedene Menschen auf unterschiedliche Art motivieren. Nicht jeder Mensch ist für die gleichen Aufgaben motiviert und selbst wenn, sind die Menschen nicht zwingend aus den gleichen Gründen motiviert (Mühlenhof 2018).

In der Psychologie wird zwischen extrinsischer und intrinsischer Motivation unterschieden. Von extrinsischer Motivation spricht man, wenn die Belohnung für eine Aufgabe von außerhalb kommt. Sind Menschen extrinsisch motiviert, führen sie eine Aufgabe nicht der Aufgabe willen aus, sondern wegen der Belohnung, die sie dafür bekommen. Ein einfaches Beispiel einer extrinsischen Belohnung, die Leute motivieren kann, ist der monetäre Lohn, den sie für eine Arbeit erhalten (De Luca 2017). Von intrinsischer Motivation spricht man, wenn eine Aufgabe wegen eines inneren Anreizes ausführt wird, der in der Tätigkeit selbst steckt. Wenn Menschen also eine Aufgabe gerne ausführen, weil sie Spaß macht, für sinnvoll erachtet wird, eine Herausforderung darstellt oder einfach interessiert (Mühlenhof 2018). Bei der Arbeit kommen typischerweise beide Formen der Motivation vor, allerdings gibt es zunehmend Bemühungen, den Anteil an intrinsischer Motivation bei der Arbeit zu erhöhen.

\subsection{Gamification}

Spiele können Menschen für eine lange Zeit fesseln. Sie können das kreative Potenzial fördern und bedeutsame Beziehungen zwischen Menschen aufbauen (Chou 2016). Die meisten Menschen betrachten Spiele jedoch hauptsächlich als Freizeitaktivität. Etwas, das wir freiwillig machen und an dem wir Spaß haben. Gamification beschäftigt sich 
damit, die Konzepte, die Spiele so vergnüglich und spannend machen, in einen spielefremden Kontext zu implementieren. Das folgende Kapitel beschäftigt sich mit der detaillierten Definition von Gamification und damit, wie Gamification eingesetzt werden kann. Auf die einzelnen Treiber hinter den Spielen und damit auch hinter Gamification wird anschließend in Zusammenhang mit dem Octalysis-Framework eingegangen.

\subsubsection{Definition Spiele}

Im Gegensatz zum Deutschen unterscheidet man im Englischen sprachlich zwischen „Game“ und „Play“. Dabei bezeichnet „Game“ vor allem das regelbasierte Spiel; „Play“ wird dagegen eher für Spiele genutzt wird, die mit wenigen oder gar keinen Regeln auskommen (Engelhard 2014). Im Rahmen dieser Arbeit wird ausschließlich auf den „Game“-Aspekt von Spielen eingegangen. McGonigal (2012) definiert hierfür vier Charakteristiken, die in sämtlichen Spielen vorhanden sind: Ein Ziel, Regeln, ein Feedbacksystem und Freiwilligkeit. Eine ergänzende Definition liefert Adams (2014), worin Spiele definiert werden als ,eine Form der Aktivität, die in einem Kontext einer vorgetäuschten Realität stattfinden und in der Teilnehmende versuchen, wenigsten ein willkürliches, nichttriviales Ziel zu erreichen, indem sie sich gemäß bestimmter Regeln verhalten." Zusammenfassend lässt sich also festhalten, dass Spiele nicht inhaltlich definiert werden, sondern über den Prozess und den dabei geltenden Rahmenbedingungen.

\subsubsection{Definition Gamification}

Der Begriff Gamification wurde in den letzten Jahren von diversen Autoren mit unterschiedlichen Definitionen verwendet. Der Begriff selbst setzt sich aus den Wörtern „Game“, Englisch für Spiel, und dem Suffix ,,-ification“, der Prozess etwas zu werden (Wiktionary 2018), zusammen. Eine mögliche Übersetzung wäre also in etwa: „Der Prozess, wie etwas zum Spiel wird“. Ungefähr 2008 ist der Begriff erstmals in den digitalen Medien aufgetaucht. Erst 2010, nachdem diverse Firmen begannen den Begriff zu verwenden, wurde ihm jedoch wirklich Beachtung geschenkt (Deterding et al. 2011). Eine der ersten Definitionen stammt von Deterding et al. (2011): „Gamification ist der Einsatz von Spiele-Elementen in einem Nicht-Spiele-Kontext.“ Dies ist bis heute eine der am weitesten verbreiteten Definitionen und wird beispielsweise im Wiktionary in ähnlicher Form verwendet: „Der Einsatz von Spielmechaniken in Nicht-Spiel-Anwendungen“ (Wiktionary 2017). Eine etwas weiterführende Definition schlug Kapp (2012) vor: „Gamification verwendet Spiele-basierte Mechanismen, Ästhetik und Spiellogik um Menschen anzusprechen, Handlungen zu motivieren, Lernen zu fördern und Probleme zu lösen“. Bei dieser Definition wurde gezielt der Teil „Nicht-Spiele-Kontext“ ausgelassen. Dies, weil frühere Autoren nie eine konkrete Definition dazu liefern konnten, was denn nun genau ein Nicht-Spiele-Kontext alles umfasst. Neu an dieser Definition ist 
dafür der Begriff der Ästhetik in diesem Zusammenhang, was erstmals impliziert, dass Gamification ansprechende Grafiken einsetzt und positive wahrgenommene Nutzererlebnisse vermittelt. Ebenfalls hinzugekommen ist „Menschen anzusprechen, Handlungen zu motivieren“, was zwei Schlüsselziele der Gamification in die Definition mit einschließt, nämlich, wie es gelingt, dass sich Leute beteiligen und engagieren und dass sie motiviert werden, eine bestimmte Aktion auszuführen (Engelhard 2014). Yu-kay Chou (2016) definiert den Begriff wie folgt: „Aus meiner Sicht ist Gamification die Kunst Spaß zu vermitteln und Elemente zu nutzen, die typischerweise in Spielen vorkommen, und diese auf produktive Handlungen in der Realität anzuwenden. Diesen Prozess meine ich, wenn ich von ,Human-Focused Design“ spreche.“ Etwas ausführlicher bedeutet diese Definition, Mechaniken aus Spielen mit Motivationspsychologie sowie Verhaltensökonomie zu verbinden und diesen auf einer technologischen Plattform ein ansprechendes User Interface zu geben. Für Chou (2016) ist eine Gamification-Maßnahme zudem im besten Fall für den Nutzer so gut wie unsichtbar. Er vergleicht dies mit einer Türklinke. Sie ist da und alle benutzen sie unbewusst mehrmals täglich, ihr wird jedoch nur selten spezifisch Beachtung geschenkt.

Chou gibt zu bedenken, dass Gamification eine Form der Manipulation ist. Diesen Einwand gilt es jedoch zu relativieren. Erstens sind Menschen ständigen Beeinflussungen ausgesetzt und nahezu jede Handlung wird bewusst oder unbewusst durch Interventionen geleitet. Zweitens sollten Gamification-Maßnahmen stets freiwillig und transparent sein. Benutzende sollten also die Gamification klar erkennen und sich dadurch nicht negativ beeinflusst oder ausgetrickst fühlen (Chou 2016).

\subsubsection{Human Centered Design}

In Gamification-Theorien wird häufig das Konzept des Human Centered Designs erwähnt. Es geht dabei darum, eine Maßnahme zu entwickeln, bei der Mensch als Nutzer im Mittelpunkt steht (Norman 2013). Human Centered Design stellt die Bedürfnisse von Nutzern stets über die Bedürfnisse der Unternehmung oder des Urhebers der Gamification-Maßnahme. Es geht darum, ob und wieso Nutzer eine bestimmte Funktion brauchen. Bei jeder Design-Entscheidung sollte man sich deshalb fragen: Wie profitieren Nutzer von diesem System (Nicholson 2012)? Ein wichtiger Bestandteil davon ist es, Gamification-Maßnahmen für Nutzer transparent zu machen. Diese sollen sehen, was genau mit der Maßnahme bezweckt wird und nicht nur, wie viele Punkte erreicht wurden, ohne zu wissen, was der Sinn dieser Maßnahme ist. Die meisten Maßnahmen, die lediglich ein Punktesystem oder eine Rangliste präsentieren, und so die extrinsische Motivation von Benutzenden ansprechen, folgen deshalb nicht den Prinzipien des Human Centered Designs (Nicholson 2012). 


\subsubsection{Gamification in Unternehmen}

Bei der Umsetzung von Gamification in internen Prozessen von Unternehmen gibt es einige wichtige Punkte zu beachten. Hier geht es beispielsweise nicht darum, jemanden zu einem Impulskauf zu überzeugen, den er kurz später bereits wieder bereut (,Buyer's Remorse“). Vielmehr steht die Langzeitmotivation der Mitarbeitenden im Fokus. Von zentraler Wichtigkeit ist, zuerst das Bedürfnis der Mitarbeitenden zu identifizieren. Man sollte die generelle Akzeptanz der Benutzer gegenüber einer solchen Maßnahme sowie deren Präferenzen kennen (Dale 2014). Dabei gilt es, die verschiedenen Spielertypen zu beachten und sicherzustellen, dass sich möglichst alle in der Maßnahme wiederfinden (Bartle 1996). Bereits hier sollte auch an die verschiedenen zeitlichen Phasen einer Gamification-Maßnahme gedacht werden (Stieglitz 2015). Außerdem sollte auch die bestehende Unternehmenskultur berücksichtigt werden (Dale 2014). Ebenfalls notwendig ist die Analyse der vorhandenen IT-Infrastruktur, da die meisten Gamification-Maßnahmen IT-gestützt sind. Da viele Gamification-Experten eine ansprechende Benutzeroberfläche empfehlen, sollte unbedingt im Voraus abgeklärt werden, ob die geplanten Maßnahmen überhaupt entsprechend umgesetzt und unterstützt werden können (Stieglitz 2015). Des Weiteren wird empfohlen, die Gamification-Maßnahme an einen geeigneten Prozess zu koppeln und sicherzustellen, dass die Mechaniken und die Ansätze, die mit der Maßnahme verfolgt werden, unmissverständlich klar sind. Ist die Maßnahme dann einmal implementiert, sollte sie wie jeder Prozess regelmäßig überprüft und, wenn nötig, angepasst werden. Eine Beurteilung der Maßnahme im Laufe der Zeit ist deshalb anzuraten, da die Maßnahmen teilweise nur kurzfristige Erfolge verzeichnen und nach einer gewissen Zeit durch die Benutzer als bedeutungslos oder gar störend wahrgenommen werden können (Stieglitz 2015).

\subsubsection{Warum Gamification?}

Warum sollte ein Unternehmen Gamification einsetzten? Warum nicht eine andere, konventionelle, bewährte Methode verwenden? Die Idee eine Arbeit durch Spielelemente interessanter $\mathrm{zu}$ machen, ist gar nicht so neu. Bereits 1984 stellte Charles Coonradt (2012) in seinem Werk „The Game of Work“ die Frage, warum Menschen für Hobbys oder im Sport bezahlen um noch mehr oder noch härter arbeiten zu dürfen, während sie für das Ausführen ihrer Arbeit einen Lohn als Motivator verlangen. IT-gestützte Spiele waren damals aber noch eine Randerscheinung und die mit ihnen einhergehenden Konzepte den Menschen weniger vertraut. Heutzutage sind Videogames zum Massenmedium geworden. Im Jahr 2012 war das Durchschnittsalter der Gamer in den USA 35 Jahre. Jeder vierte davon war über 50 Jahre alt. Außerdem spielten dort $97 \%$ aller Jugendlichen irgendeine Art von Games (McGonigal 2012). Durch die Spiele werden die Benutzer gefesselt und angeregt. Viele können den Feierabend kaum erwarten, wenn sie sich endlich wieder ihren Spielen widmen können. 
Volkswagen generierte 33 Mio. Aufrufe auf ihrer Website durch die Gamification-Maßnahme Perfect Car und erhielt dadurch 119.000 neue Ideen für Autos. Im Spiel Foldit, in dem Spieler neue Proteine designen können, halfen 240.000 registrierte Benutzer eine fehlende Verbindung in der Aids-Forschung zu finden. Ein Problem, an dem Forscher 15 Jahre lang gearbeitet hatten, konnte von Gamern in nur 10 Tagen gelöst werden. Eine Lernplattform für Studenten, die fürs GMAT lernten, konnte mit Gamification-Maßnahmen die Zeit, die die Studierenden darauf verbrachten und versuchten ihr Testresultat zu verbessern, um 370 \% erhöhen. Des Weiteren gibt die US Armee, deren Zielgruppe vor allem junge Männer sind, heute mehr Geld für Rekrutier-Spiele aus, als für jede andere Werbeplattform (Chou 2016). Dies sind nur ein paar Beispiele, wie Gamification in allen möglichen Situationen zu enormen Erfolgen führen kann. Somit dürfte deutlich geworden sein: Gamification ist längst keine belächelte Randerscheinung mehr, sondern ein Business mit enormem Potenzial, das im Zuge der digitalen Transformation mehr und mehr genutzt werden wird.

\subsection{Das Octalysis-Framework}

Das Octalysis-Modell ist ein Modell, das dazu dient, gamifizierte Maßnahmen zu planen, zu gestalten, zu analysieren oder zu verbessern. Das Modell wurde vom Gamification-Experten Yu-kai Chou empirisch anhand seiner eigenen Erfahrungen mit Spielen entwickelt und später mit wissenschaftlichen Theorien ergänzt und begründet. Aufgrund des fehlenden psychologischen Hintergrundes nennt Chou das Modell auch einen „Framework" und nicht psychologische Studie oder Theorie (Chou 2016). Dennoch ist es aus Anwendungssicht nützlich und wird auch in akademischen Kreisen als solide Grundlage für kulturelle Interventionen diskutiert.

Das Modell baut auf den sogenannten Core Drives auf, die verschiedenen Motivatoren hinter einer Aktion repräsentieren (Abb. 4.1). Die Core Drives stellen dabei theoretische Treiber dar, die Nutzer dahingehend motivieren, eine gewisse Aktion auszuführen. Chou (2016) weist dabei darauf hin, dass diese Core Drives nicht nur in Spielen, sondern in sämtlichen menschlichen Handlungen eine Rolle spielen. Fehlen die Core Drives komplett, ist keine Motivation vorhanden und es kommt infolgedessen keine Aktion zustande. Die Spieleindustrie ist lediglich die erste Industrie, die die Core Drives soweit gemeistert hat, dass sie Nutzer gezielt über viele Stunden motivieren können (Chou 2016).

Hauptziel des Octalysis-Modells ist es, Motivatoren zu identifizieren oder aufzuzeigen, wie zusätzliche Motivation für eine Aktion angeregt werden kann. Dabei sollte stets darauf geachtet werden, wie sich der Nutzer fühlt und weniger, wie man Gamification-Elemente in eine Aktion integrieren kann. Zudem sollte man es vermeiden, sich beim Planen von Maßnahmen von Spielen beeinflussen zu lassen, sondern sich stattdessen darauf konzentrieren, wie und welche der acht Core Drives in eine Maßnahme implementiert werden können. Dazu gibt es zu jedem Core Drive mehrere Gamification-Techniken, die speziell 


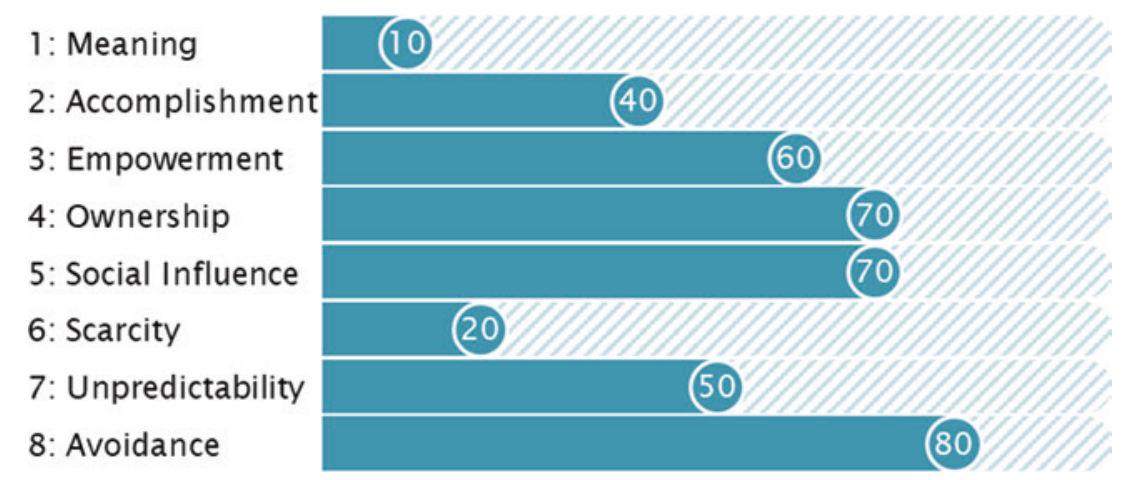

Abb. 4.1 Visualisierung des Octalysis-Modells zur Gestaltung und Analyse von gamifizierten Maßnahmen. (Illustratives Beispiel; eigene Darstellung)

dafür gedacht sind, diesen Core Drive in einem Arbeitsumfeld effizient umsetzen zu können (Chou 2016; aus Platzgründen wird in dieser Arbeit nicht auf die einzelnen Gamification-Techniken eingegangen, diese finden sich im Internet und in der Originalliteratur).

Durch das Gewichten sämtlicher Core Drives auf einer Skala von 0 bis 100 bezüglich einer Maßnahme kann diese bewertet werden; innerhalb des Modells wird dies als Octalysis-Score bezeichnet. Da theoretisch jede Aktion die Core Drives in einem geringen Maß beinhaltet, ist ein Score von weniger als 50 kein Indiz, dass hier spezielle Gamification-Techniken angewandt werden. Der Score ist außerdem als absolute Zahl wenig aussagekräftig, da die Gewichtung der Maßnahmen subjektiv ist. Anstatt damit eine Maßnahme als ,gut“ oder ,schlecht“ zu beurteilen, sollte er eher dafür benutzt werden, zu identifizieren, wo die relativen Stärken liegen und wo noch Potenzial für Verbesserungen vorhanden ist (Chou 2016).

\subsubsection{Octalysis Design Levels}

Das Octalysis-Modell ist in der bisherigen Ausarbeitung auf drei Levels ausgelegt: Core Drives, Spielephasen und Spielertypen (Tab. 4.1). In den meisten Situationen ist jedoch bereits das erste Level ausreichend (Core Drives), um eine Maßnahme bezüglich Gamification zu analysieren oder zu entwickeln (Chou 2012). Für die Studie wurde deshalb nur der erste Level zur Analyse der kulturverändernden Maßnahmen verwendet.

Der erste Level des Octalysis-Frameworks beinhaltet das namensgebende Oktagon mit den acht Core Drives. Aus Gründen der besseren Lesbarkeit wurde die ursprünglich achteckige Visualisierung als Balkendiagramm linearisiert. Es zeigt auf, welche Faktoren die Motivatoren bei Spielen sind (Tab. 4.2). Für die Auswertung einer bestehenden Maßnahme wird analysiert, welcher Core Drive darin in welchem Ausmaß vorhanden 
Tab. 4.1 Die drei konzeptionellen Level im Octalysis-Modell. (angelehnt an Chou (2016))

\begin{tabular}{l|l|l}
\hline Level & Konzept & Beschreibung \\
\hline 1. & Core Drives & $\begin{array}{l}\text { 4 Faktoren, die als Motivatoren bei Spielen wirken: Epic Meaning \& } \\
\text { Calling, Development \& Accomplishment, Empowerment of Creativity } \\
\text { \& Feedback, Ownership \& Possession, Social Influence \& Relatedness, } \\
\text { Scarcity \& Impatience, Unpredictability \& Curiosity, Loss \& Avoidance }\end{array}$ \\
\hline 2. & Spielephasen & $\begin{array}{l}\text { Phasen, die Spieler über den Gesamtverlauf eines Spiels durchleben: } \\
\text { Discovery, Onboarding, Scaffolding, Endgame }\end{array}$ \\
\hline 3. & Spielertypen & Achievers, Explorers, Socializers, Killers \\
\hline
\end{tabular}

ist. Anschließend wird jedem Core Drive eine entsprechende Zahl zwischen 0 und 100 zugeordnet. Aus den Einschätzungen ergibt sich ein Profil der untersuchten Maßnahme.

\subsubsection{White Hat and Black Hat Gamification}

Das Octalysis-Framework wird in White-Hat-Gamification- und Black-Hat-Gamification-Maßnahmen unterteilt (Tab. 4.3). In der Visualisierung des Modells sind dabei die oberen drei Core Drives White-Hat- und die untersten drei Black-Hat-Maßnahmen (Abb. 4.2). Die Core Drives vier und fünf können je nach Anwendung sowohl White-Hat- wie auch Black-Hat-Einflüsse haben, sind jedoch für keines davon typisch. Black-Hat-Elemente sind nicht typischerweise schlecht und White Hat gut. Für den Erfolg einer Maßnahme braucht es oft Einflüsse von beiden Typen. Die Black Hat Core Drives beinhalten jedoch diejenigen Maßnahmen, die, über einen längeren Zeitraum angewendet, eher zu Frust oder Abhängigkeit führen können. Deshalb sollte man beim Entwickeln einer Gamification-Maßnahme auch zuerst die White Hat Core Drives betonen (Chou 2016).

White Hat Core Drives sind die Elemente, die für eine Langzeitmotivation sorgen und zufriedenstellend wirken. Sie geben uns das Gefühl, die Kontrolle über unser eigenes Leben und unsere Aktionen zu haben. Hat eine Maßnahme keine White-Hat-Elemente, ist die Chance groß, dass sie langfristig nicht erfolgreich ist. Die große Schwäche der White Hat Core Drives ist, dass sie kein Gefühl der Dringlichkeit im Benutzer auslösen. Deshalb scheitern Maßnahmen, die sich nur darauf konzentrieren, meist bereits in den Discovery- und Onboarding-Phasen. Beim Arbeiten in einem Unternehmen, in welchem diese beiden Phasen nach der Anstellung jedoch sehr rasch überwunden sind und es um eine Langzeitverbindung geht, sollte man sich vorwiegend auf White-Hat-Maßnahmen fokussieren. Dies führt dazu, dass sich Mitarbeitende gut fühlen, mit dem Unternehmen wachsen können und auch länger dabeibleiben (Chou 2016).

Dies deckt sich mit der Self-Determination-Theorie von Deci (2008) bzw. deren Interpretation durch Pink (2011). Diese besagt, dass Menschen insbesondere durch drei intrinsische Treiber motiviert werden: Erstens Autonomie, das Bedürfnis selbst 
Tab. 4.2 Die acht Faktoren, die als Motivatoren bei Spielen wirken. (angelehnt an Chou (2016))

\begin{tabular}{|c|c|c|}
\hline $\mathrm{Nr}$. & Core Drive & Erklärung \\
\hline 1. & Epic Meaning \& Calling & $\begin{array}{l}\text { Dieser Core Drive wirkt, wenn jemand das } \\
\text { Gefühl hat, Teil von etwas Größerem, Wich- } \\
\text { tigerem als sich selbst zu sein, oder zu etwas } \\
\text { Bestimmten berufen zu sein }\end{array}$ \\
\hline 2. & Development \& Accomplishment & $\begin{array}{l}\text { Dieser Core Drive spricht die innere Moti- } \\
\text { vation an, Fortschritte zu erzielen und die } \\
\text { eigenen Fähigkeiten zu verbessern um im } \\
\text { Endeffekt eine Herausforderung überwinden } \\
\text { zu können }\end{array}$ \\
\hline 3. & Empowerment of Creativity \& Feedback & $\begin{array}{l}\text { Hier geht es darum, den Benutzer immer } \\
\text { wieder einen kreativen Prozess durchlaufen } \\
\text { zu lassen, ihn zu ermutigen, Neues auszu- } \\
\text { probieren und ihm eine unmittelbare Rück- } \\
\text { meldung dazu zu geben. Dieser Core Drive } \\
\text { beinhaltet was die meisten Menschen unter } \\
\text { „spielen“ verstehen }\end{array}$ \\
\hline 4. & Ownership \& Possession & $\begin{array}{l}\text { Hier geht es darum den Benutzern das Gefühl } \\
\text { zu geben, etwas zu besitzen oder zu kontrol- } \\
\text { lieren. Entscheidungen werden hier vor allem } \\
\text { analytisch getroffen und Besitztum ist der } \\
\text { primäre Motivationsfaktor }\end{array}$ \\
\hline 5. & Social Influence \& Relatedness & $\begin{array}{l}\text { Core Drive } 5 \text { beinhaltet sämtliche sozialen } \\
\text { Elemente einer Maßnahme, wie Mentoring, } \\
\text { soziale Akzeptanz, soziales Feedback, Gesell- } \\
\text { schaft, Rivalität, der Wettbewerb mit anderen } \\
\text { Menschen oder auch Neid. Grundsätzlich } \\
\text { zählen dazu sämtliche Aktivitäten, bei denen } \\
\text { es darum geht, wie sich andere Menschen } \\
\text { uns gegenüber verhalten und was sie über uns } \\
\text { denken oder sagen }\end{array}$ \\
\hline 6. & Scarcity \& Impatience & $\begin{array}{l}\text { Dieser Core Drive kommt zum Zug, wenn wir } \\
\text { etwas haben wollen, einfach nur, weil es sel- } \\
\text { ten, nicht sofort erreichbar oder das Erreichen } \\
\text { mit großen Schwierigkeiten verbunden ist. } \\
\text { Menschen haben eine natürliche Tendenz } \\
\text { dazu, zu wollen, was sie nicht haben können. } \\
\text { Exklusivität zieht Menschen an und bringt sie } \\
\text { dazu, mehr für etwas zu tun oder zu bezahlen }\end{array}$ \\
\hline 7. & Unpredictability \& Curiosity & $\begin{array}{l}\text { Dieser Core Drive beinhaltet alles was mit } \\
\text { Zufällen oder Chancen sowie mit Situationen, } \\
\text { bei denen man nicht genau weiß, was als } \\
\text { Nächstes passiert zu tun hat }\end{array}$ \\
\hline
\end{tabular}


Tab. 4.2 (Fortsetzung)

\begin{tabular}{l|l|l}
\hline Nr. & Core Drive & Erklärung \\
\hline 8. & Loss \& & Menschen tendieren dazu, etwas, das man \\
& Avoidance & $\begin{array}{l}\text { bereits erreicht hat, nicht mehr verlieren zu } \\
\text { wollen oder verhindern zu wollen, dass etwas } \\
\text { Schlechtes eintritt }\end{array}$ \\
\hline
\end{tabular}

Tab. 4.3 Einteilung in White-Hat- und Black-Hat-Gamification. (angelehnt an Chou (2016))

\begin{tabular}{l|l|l|l}
\hline White Hat & \multicolumn{3}{l}{ Black Hat } \\
\hline Core Drive 1 & Epic Meaning \& Calling & Core Drive 6 & Scarcity \& Impatience \\
\hline Core Drive 2 & $\begin{array}{l}\text { Development \& Accomplish- } \\
\text { ment }\end{array}$ & Core Drive 7 & Unpredictability \& Curiosity \\
\hline Core Drive 3 & $\begin{array}{l}\text { Empowerment of Creativity \& } \\
\text { Feedback }\end{array}$ & Core Drive 8 & Loss \& Avoidance \\
\hline Right Brain & \multicolumn{2}{l}{ Left Brain } & \\
\hline Core Drive 3 & $\begin{array}{l}\text { Empowerment of Creativity \& } \\
\text { Feedback }\end{array}$ & Core Drive 2 & $\begin{array}{l}\text { Development \& Accomplish- } \\
\text { ment }\end{array}$ \\
\hline Core Drive 5 & Social Influence \& Relatedness & Core Drive 4 & Ownership \& Possession \\
\hline Core Drive & Unpredictability \& Curiosity & Core Drive 6 & Scarcity \& Impatience \\
\hline
\end{tabular}

White Hat

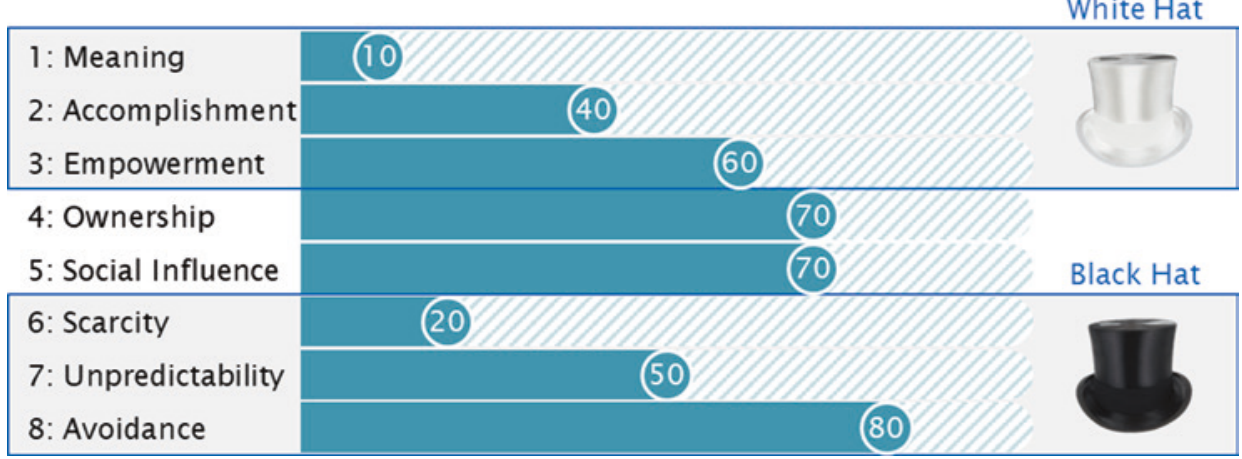

Abb. 4.2 Einteilung der Core Drives in White-Hat- und Black-Hat-Motivatoren. (Eigene Darstellung)

verantwortlich zu sein und selbst entscheiden zu können was sie machen. Zweitens die Meisterschaft einer Disziplin, also seine Fähigkeiten verbessern zu können und stetig dazu zu lernen. Drittens, einen Sinn zu sehen, in dem was sie tun. Diese Motivatoren finden sich alle in den White Hat Core Drives wieder. 


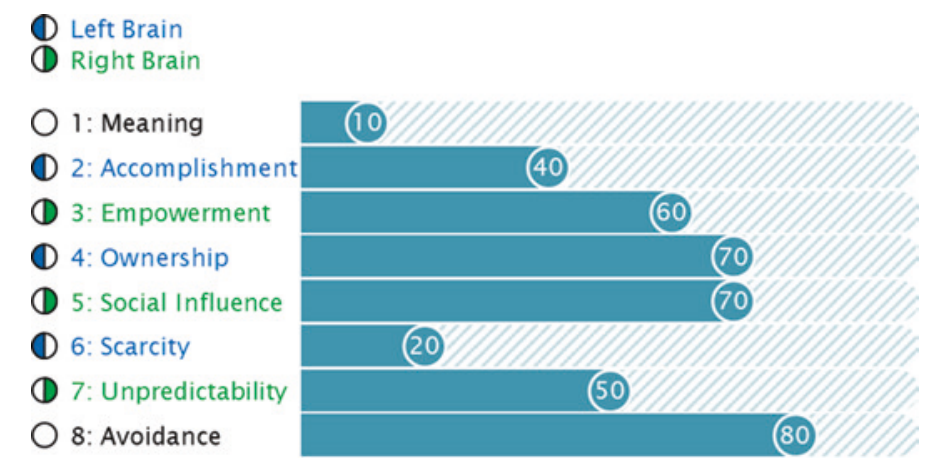

Abb. 4.3 Einteilung der Core Drives in Right-Brain- und Left-Brain-Motivatoren. (Eigene Darstellung)

Black Hat Drives führen in der Regel zu größeren kurzfristigen Erfolgen, indem sie den Menschen ein deutliches Gefühl der Dringlichkeit vermitteln. Damit können sie gerade zu Beginn einer Maßnahme sehr erfolgreich eingesetzt werden. Wie bereits erwähnt heißt Black Hat nicht, dass diese Elemente automatisch schlecht sind. Viele Menschen setzten sich freiwillig Black-Hat-Beeinflussungen aus, um sich selbst für etwas zu motivieren. Diese Core Drives haben das Potenzial, rasch viele Menschen zu mobilisieren. Im Endgame fallen diese dann jedoch oft ab und können, wenn man ihnen zu lange und in zu großen Dosen ausgesetzt ist, gar einen gegenteiligen Effekt haben und zu Frust oder auch zu Suchtverhalten führen. Dies kommt oft mit dem Gefühl, die eigenen Aktionen nicht mehr selbst unter Kontrolle zu haben und hinterlässt deshalb oft einen fahlen Beigeschmack. Da Black-Hat-Elemente oft rasche und zahlengetriebene Resultate liefern, werden in der Industrie vornehmlich diese angewandt. Insbesondere im Verkauf, wo es darum geht, dass jemand einen Kaufvorgang erfolgreich abschließen soll, funktionieren sie gut. Die Langzeitmotivation spielt in diesem Szenario meist keine Rolle. Hat eine Maßnahme gar keine Black-Hat-Elemente, besteht zudem das Risiko, dass sie gar nicht entdeckt wird und deshalb nie die Chance bekommt ein durchschlagender Erfolg zu werden (Chou 2016).

Neben der Einteilung nach Black Hat und White Hat wird das Octalysis-Framework in Right Brain und Left Brain Gamification unterteilt (Abb. 4.3). Die Core Drives 1 und 8 gehören dabei weder zum einen noch zum anderen. Die Right-Brain- und Left-Brain-Terminologie des Octalysis-Frameworks hat nichts mit dem physikalischen Gehirn und dessen zwei Hälften zu tun. Die Begriffe wurden symbolisch gewählt, um aufzuzeigen, welche Core Drives eher vom logischen Denken beeinflusst werden und welche eher vom emotionalen. Dabei fokussieren sich die Right Brain Core Drives insbesondere auf intrinsische Motivation während bei den Left Brain Core Drives die extrinsische Motivation im Vordergrund steht. Left Brain Core Drives sind dabei typischerweise einfacher zu entwickeln, weshalb sie in der Arbeitswelt auch viel häufiger genutzt werden. Es ist 
viel einfacher, einer Aktivität einen Preis zuzuordnen, als die Aktivität selbst spannend zu gestalten (Chou 2016). Für die Gestaltung von Gamification-Maßnahmen sollten jedoch beide Gruppen berücksichtigt werden.

Die Charakteristika von Right Brain Core Drives sind Kreativität, soziale Aspekte und Neugier. Dies korrespondiert mit intrinsischen Motivatoren, die einen Menschen antreiben. Intrinsische Motivation kommt davon, wenn einem eine Aufgabe positive Gefühle auslöst und man diese für sich selbst macht und nicht, weil jemand einem eine Belohnung dafür gibt. Right Brain Core Drives sind von Natur aus erfahrungsorientiert und eher auf den Prozess fokussiert als auf das Resultat (Chou 2016). Die drei Right Brain Core Drives finden sich auch in den Forschungsarbeiten von Jane McGonigal (2012) wieder, in der die wichtigsten intrinsischen Belohnungen, um glücklich zu sein, folgendermaßen beschrieben werden: eine befriedigende Arbeit, das Gefühl, im Laufe der Zeit besser zu werden, soziale Kontakte pflegen sowie das Gefühl, Teil von etwas Größerem zu sein.

Left Brain Core Drives haben vor allem mit Logik, mit Eigentum und mit analytischem Denken zu tun. Sie korrespondieren mit extrinsischen Motivationsfaktoren. Extrinsische Motivation kommt von einem Ziel, einem Zweck oder einer Belohnung, die uns jemand anderes gibt, und fokussieren auf Resultate. Wir erledigen eine Aufgabe, die nicht interessant ist, unter Umständen trotzdem, wenn uns jemand eine Belohnung dafür gibt. Dies kann dabei helfen Leute für eine monotone Arbeit zu begeistern oder um das initiale Interesse von jemandem zu gewinnen. Extrinsische Belohnungen können aber auch gefährlich sein. Hat eine Arbeit nur extrinsische Anreize, machen wir sie oft so rasch wie möglich oder so, dass sie gerade noch gut genug ist um die extrinsische Belohnung zu erhalten (Chou 2016). Dies deckt sich auch mit psychologischen Studien, die im Bereich der Arbeitsentlohnung gemacht wurden. Viele Vorgesetzte in Unternehmen denken, dass Mitarbeitende besser arbeiten, wenn man sie besser bezahlt. Dies funktioniert zwar bei monotonen oder mechanischen Arbeiten. Bei konzeptionellen Arbeiten wirkt dies jedoch nur kurzfristig. Jemand mag eine Arbeit einer anderen vorziehen, weil er für diese besser bezahlt wird. Langfristig macht man ohne intrinsische Motivation seine Arbeit hingegen nur gut genug, um seinen Job nicht $\mathrm{zu}$ verlieren (Pink 2011). Schlimmer noch: egal ob Geld, Schulnoten, Beförderungen, Aufmerksamkeit oder andere materielle Belohnungen, Forscher sind sich einig, dass extrinsischen Belohnungen nachzueifern, ein sicherer Weg ist, die eigene Freude zu sabotieren. Zudem kann das Hinzufügen von extrinsischen Belohnungen zu einer Tätigkeit, die wir freiwillig verrichten sogar gegenteilige Auswirkungen haben. Oft ist Leuten eine Tätigkeit verleidet, nachdem sie dafür eine Bezahlung erhalten haben, da sie nun das Gefühl bekamen, ihre Zeit sei mehr wert oder sie haben den Spaß verloren, da die Tätigkeit nun nicht mehr freiwillig war, sondern für die Bezahlung erledigt werden musste (McGonigal 2012). 


\subsection{Case Study und Methodik}

Im folgenden Kapitel wird die Case Study eingeführt und die Methodik zur Erhebung der empirischen Daten für diese Untersuchung erörtert. Es wird dabei zuerst die Methode für die Abbildung der bestehenden Maßnahmen der zu untersuchenden Firma festgelegt. Anschließend wird die Methode für die Interviews zur Beantwortung der Zielsetzungen erklärt.

\subsubsection{Charakterisierung des Unternehmens der Case Study}

Die Firma Acme Apps AG wurde 2010 gegründet. Sie spezialisierte sich darauf, benutzerfreundliche digitale Produkte zu entwickeln, die das tägliche Leben der Benutzer vereinfachen. Dabei decken sie die gesamte Wertschöpfungskette ab, von der ersten Idee über User Research, Konzept und Design bis hin zur technischen Entwicklung und dem Marketing. Das Konzept war ein Erfolgsmodell und die Acme Apps $A G$ wuchs rasch. Heute beschäftigt die Firma rund 30 Personen an ihrem Sitz in einer Schweizer Großstadt. Noch immer entwickeln sie Apps und weitere digitale Produkte, haben ihr Portfolio jedoch auch auf Workshops, das Gestalten von Benutzeroberflächen und interaktiven Erlebnissen sowie systematischer App-Vermarktung erweitert.

\subsubsection{Maßnahmen zur Kulturveränderung}

Unternehmenskultur wird bei Acme Apps AG großgeschrieben. „Kultur ist König“ steht dann auch als erste Überschrift auf der Website der Firma. Für den Unternehmensgründer ist die Kultur in seinem Unternehmen das wertvollste Gut. Da die Arbeit ein bedeutender Teil des Lebens ist, soll diese den Mitarbeitenden ein gewisses Maß an Zufriedenheit bereiten und einen Gewinn darstellen; nicht nur finanziell, sondern auch menschlich und geistig. So soll die Unternehmenskultur die Mitarbeitenden motivieren, unterstützen und glücklicher machen. Die Firma Acme Apps AG strebt dabei eine Kultur auf Augenhöhe an, die durch Respekt, Toleranz und Mitbestimmung getragen wird. Deshalb sollen auch alle ihre Bedürfnisse mitteilen können und ernst genommen werden. Um dies sicherzustellen, hat Acme Apps AG die traditionelle Geschäftsleitung abgeschafft und die meisten Entscheidungen werden nun in verschiedenen Boards getroffen, an welchen alle Mitarbeitenden teilnehmen können. Eines davon ist das Kultur-Board, in dem auch die drei Maßnahmen zur Kulturbeeinflussung entwickelt wurden: „Time to Play“, „Abzeichen \& Ehrenmedaillen“ sowie „Ämtlikarten“, die nachfolgend kurz skizziert werden. 


\subsubsection{Time to Play}

Im Büro von Acme Apps $A G$ hat es diverse Spielemöglichkeiten wie Ping Pong, Darts oder Tischfußballspiel. Diese wurden aufgestellt, da kurze Spiele während der Arbeitszeit als wichtige Ablenkung und Entspannung angesehen werden. Die Mitarbeitenden sollten die Möglichkeit haben, kurz aufzustehen und den Kopf durchlüften zu können. Diese kurze Ablenkung und der geförderte soziale Kontakt sollen die Arbeitsqualität verbessern. Da immer jemand das Spielen initiieren und Mitspielende mobilisieren musste, wurden die Möglichkeiten jedoch selten genutzt. Um dies zu ändern, wurde im Kultur-Board beschlossen, einen digitalen Zufallsgenerator zu erstellen. Dieser geht zweimal in der Woche während der Arbeitszeit los und fordert sämtliche Mitarbeitenden auf, für zehn Minuten gemeinsam zu spielen. Dank dieser Maßnahme wird nun regelmäßig gespielt, der Kopf gelüftet, Bewegung initiiert und der soziale Kontakt in einem anderen Rahmen gepflegt.

\subsubsection{Abzeichen und Ehrenmedaillen}

Acme Apps $A G$ hat es sich zum Ziel gesetzt, faire Löhne zu zahlen und transparente Lohnkriterien dafür anzuwenden. Außerdem sollen Lohnverhandlungen gänzlich abgeschafft werden. Dies wurde durch die Entwicklung eines eigenen Lohnsystems umgesetzt. So basiert der Lohn für jeden Mitarbeitenden nun auf genau festgelegten Kriterien, wie die absolvierte Ausbildung, Facherfahrung im entsprechenden Arbeitsgebiet, Firmentreue und Verantwortung. Bewusst hat man sich dabei gegen eine individuelle Bewertung der Leistung als Lohnfaktor entschieden. Die Hauptgründe hierfür waren, dass die erbrachte Leistung oft nur subjektiv einschätzbar ist und die Zusammenarbeit im Team gefördert werden soll. Um sowohl die Firmentreue noch weiter zu fördern, aber auch besondere Verdienste doch auf eine Weise zu würdigen, wurde ein Achievementsystem ins Leben gerufen. Dafür wurden spezielle Medaillen entwickelt und mithilfe eines 3D-Druckers gedruckt. Medaillen gibt es sowohl für Firmentreue wie auch für außerordentliches Engagement in der Projektarbeit. Die Medaillen kommen dabei in unterschiedlichen Stufen. So erhält man nach drei Jahren Firmentreue eine Bronzemedaille, nach fünf Jahren die Silbermedaille und nach sieben Jahren die Goldmedaille. Für außerordentliches Engagement bekommt man zuerst die Auszeichnung „Hirsch“, wird danach zum „Meister“, dann zum „Held“ und schlussendlich zum „Guru“ ernannt. Erhält man eine Medaille, darf man zusätzlich auch noch einen Wunsch äußern, der nach Möglichkeit erfüllt wird, oder man erhält eine kleine Bargeldprämie. Die Auszeichnungen werden den Mitarbeitenden jeweils an den Teammeetings feierlich übergeben. Das (stolze) Tragen oder Ausstellen der Medaille ist anschließend jedem selbst überlassen.

\subsubsection{3 Ämtlikarten}

Damit im Büro von Acme Apps $A G$ alles funktioniert, gibt es viele kleinere, wöchentlich anstehende Aufgaben („Ämtli“). So müssen beispielsweise die Pflanzen gegossen, das Altpapier entsorgt, die Kaffeemaschine gepflegt, aktuelle Tweets vom Firmen-Account aus getätigt oder das nächste Teammeeting geleitet werden. Ursprünglich wurden 
diese Arbeiten freiwillig von Mittarbeitenden übernommen, doch wurde es mit der Zeit immer aufwendiger, sämtliche Aufgaben zu verteilen. Deshalb wurden aufgrund eines Inputs vom Kultur-Board Karten erstellt, mit denen die Aufgaben zufällig verteilt werden konnten. Nach den ersten erfolgreichen Ziehungen wurde dann ein komplettes eigenes Kartenset entwickelt, mit allen vorhandenen Aufgaben und genügend Nieten für die restlichen Mitarbeitenden. Die Karten werden als eine einfachere Maßnahme als ein konventioneller „Ämtliplan“ angesehen, zudem wird die Maßnahme mit einer Portion Nervenkitzel angereichert. Die Ziehung ist jedes Mal ein kleines, positiv aufgeladenes Ereignis und macht allen Beteiligten Spaß. Die Aufgaben selbst werden seither nicht mehr so negativ, sondern als Teil des Spiels angesehen und von allen zuverlässig ausgeführt. Die Erledigung der Arbeiten wird jeweils vor der Ziehung in der Folgewoche kontrolliert.

\subsubsection{Interviewerhebung zur Wahrnehmung der Maßnahmen}

Um die Fragestellungen der Studie beantworten zu können, wurden strukturierte Interviews mit Mitarbeitenden von Acme Apps AG durchgeführt. Es handelte sich um qualitative Interviews, da mit diesen die kollektiven Strukturen herausgearbeitet werden sollten. Da lediglich wenige Antworten benötigt wurden, diese jedoch umso ausführlicher ausfallen sollten, wurden die Interviews schriftlich geführt. Dadurch wurde den Interviewpartnern mehr Zeit zur durchdachten Beantwortung der Fragen gegeben. Die zu erwartende niedrigere Rücklaufquote wurde dabei bewusst in Kauf genommen, da von den rund dreißig Mitarbeitenden lediglich drei bis fünf Antworten benötigt wurden. Damit die Interviewfragen ohne Vorkenntnisse der Theorie beantwortet werden konnten, wurden diese vorgängig operationalisiert. Die Interviews wurden anonymisiert und in einer zusammenfassenden Inhaltsanalyse aufgearbeitet.

\subsection{Ergebnisse}

Im folgenden Kapitel geht es darum, die Theorie mit der Praxis zu verknüpfen. Dazu wurden die drei Maßnahmen „Time to Play“, „Abzeichen \& Ehrenmedaillen“ sowie „Ämtlikarten“ ausgewertet. In einem ersten Schritt wurden diese mithilfe des Octalysis-Modells analysiert. Hierfür wurde ausgewertet, welche Core Drives sich in welchen Maßnahmen wiederfinden und wie diese konzipiert wurden (Tab. 4.4). In einem zweiten Schritt wurden anschließend die in den Interviews erhobenen Daten mit dem Framework verknüpft, um zu eruieren, ob die Mitarbeitenden die Core Drives als solche wahrnehmen. Anschließend wurde untersucht, ob die Maßnahmen tatsächlich zur Unternehmenskultur beitragen und ob sie die Motivation der Mitarbeitenden anregen.

Untersucht man die Maßnahme „Time to Play“, so fällt zunächst der Core Drive 7: 
Tab. 4.4 Ergebnisse der Interviews und Analyse der kulturverändernden Maßnahmen. (angelehnt an Chou (2016))

\begin{tabular}{l|l|l|l|l}
\hline & Core Drive & Time to Play & Abzeichen \& Ehrenmedaillen & Ämtlikarten \\
\hline 1. & Epic Meaning \& Calling & - & $\checkmark$ & $\checkmark$ \\
\hline 2. & $\begin{array}{l}\text { Development \& Accomplish- } \\
\text { ment }\end{array}$ & - & $\checkmark$ & $\checkmark$ \\
\hline 3. & $\begin{array}{l}\text { Empowerment of Creativity \& } \\
\text { Feedback }\end{array}$ & - & $\checkmark$ & $\checkmark$ \\
\hline 4. & Ownership \& Possession & - & - & $\checkmark$ \\
\hline 5. & $\begin{array}{l}\text { Social Influence \& Related- } \\
\text { ness }\end{array}$ & $\checkmark$ & $\checkmark$ & $\checkmark$ \\
\hline 6. & Scarcity \& Impatience & - & $\checkmark$ & $\checkmark$ \\
\hline 7. & Unpredictability \& Curiosity & $\checkmark$ & $\checkmark$ & $\checkmark$ \\
\hline 8. & $\begin{array}{l}\text { Loss \& } \\
\text { Avoidance }\end{array}$ & - & - & - \\
\hline
\end{tabular}

Unpredictability \& Curiosity auf. Insbesondere der Teil der Unvorhersehbarkeit ist im Design dieser Maßnahme stark verankert. Gemäß Aussagen des CEO wurde die Maßnahme gezielt so erarbeitet, dass eine „höhere Macht“ zu zufälligen Zeiten alle Mitarbeitenden zusammenbringt.

Das Miteinander und damit Core Drive 5: Social Influence \& Relatedness ist der zweite auffällige Aspekt dieser Maßnahme. Ziel davon ist es, nebst der Ablenkung und der Entspannung, insbesondere den sozialen Austausch zu fördern. Dies passiert, indem die Maßnahme alle Anwesenden zum gemeinsamen Spielen auffordert und nicht nur einzelne. Dies kann sogar Kunden oder Auftraggeber beinhalten, die sich zur Zeit des Alarms zufälligerweise im Gebäude aufhalten.

Da der Alarm lediglich zweimal in der Woche losgeht, könnte man denken, dass auch Core Drive 6: Scarcity \& Impatience in einem gewissen Masse vorhanden ist. Dies ist jedoch nicht der Fall, da die Mitarbeitenden von Acme Apps AG theoretisch jederzeit eine kurze Pause fürs Spielen einlegen können. Es finden sich in dieser Maßnahme hauptsächlich zwei Core Drives wieder. Beide korrespondieren mit intrinsischen Motivatoren (Right Brain). Dies deutet darauf hin, dass die Nutzer sich gerne freiwillig der Maßnahme aussetzen werden, da die Maßnahme selbst belohnend wirkt. Core Drive 7: Unpredictability \& Curiosity wird außerdem den Black-Hat-Gamification-Maßnahmen zugeordnet. Dies deutet darauf hin, dass durch die Maßnahme ein gewisser Sinn für Dringlichkeit entsteht. Man muss nämlich zu genau diesem Zeitpunkt aufstehen und spielen, sonst verpasst man die Chance. Dies kann zwar durchaus eine Langzeitmotivation generieren, durch die Black-Hat-Einflüsse aber über längere Zeit auch als lästig angesehen werden und die erhoffte Wirkung verlieren.

Durch die Auswertung der Interviews wird klar, dass die Ungewissheit, die Teil des Designs dieser Maßnahme ist, durch die Mitarbeitenden nicht mehr als solche angesehen 
wird. Zwar ist die Zeit, zu welcher der Alarm losgeht, noch immer zufällig, doch die Sicherheit, dass er zweimal in der Woche kommt, relativiert dies. Core Drive 7: Unpredictability \& Curiosity wird also nicht als treibendes Element dieser Maßnahme wahrgenommen. Ob dies nie der Fall war oder dieser Core Drive sich über die Zeit langsam abgebaut und an Bedeutung verloren hat, ließ sich nicht mehr ermitteln.

Anders sieht dies bei Core Drive 5: Social Influence \& Relatedness aus. Dieser wird als treibende Kraft hinter der Maßnahme angesehen und durch die Mitarbeitenden sehr geschätzt. Der zwanglose Austausch während einer anderen Aktivität als der täglichen Arbeit lässt es zu, Arbeitskollegen von einer anderen Seite kennenzulernen.

Die Maßnahme „Abzeichen \& Ehrenmedaillen“ ist eine typische Form des Core Drive 2: Development \& Accomplishment. Man erhält eine Auszeichnung, wenn man lange genug in der Firma gearbeitet oder wenn man etwas Außerordentliches geleistet hat. Dies in Form von physikalischen Medaillen zu tun, verleiht der Maßnahme noch ein bisschen Core Drive 5: Social Influence \& Relatedness. Dies einerseits durch die Art, wie sie verliehen werden, während des Teammeetings und mit Fanfaren, aber anderseits auch dadurch, dass man die Medaille später aufstellen, aufhängen oder tragen kann. Damit kann man andere stets daran erinnern, dass man die Auszeichnung erhalten hat. Weil die Auszeichnung vor dem gesamten Team vorgenommen wird, und nicht während eines Mitarbeitergesprächs, bekommen die Auszeichnung alle mit, was zur Verbundenheit beiträgt. Die Tatsache, dass nicht einfach jeder eine Medaille hat, sondern man dafür entweder schon lange dabei sein oder etwas wirklich Herausragendes geleistet haben muss, gibt der Maßnahme zudem ein bisschen von Core Drive 6: Scarcity \& Impatience. In sehr geringem Maße sind auch noch weitere Core Drives vertreten. Die Treuemedaillen haben ein bisschen etwas von einem Meilenstein, weshalb man sie Core Drive 3: Empowerment of Creativity \& Feedback zuordnen könnte. Die Projektabzeichen kommen meist unverhofft, was etwas von Core Drive 7: Unpredictability \& Curiosity hat. Da man sich aber kaum ständig fragt, wann man nun endlich eine Medaille erhält, ist das wohl nur sehr schwach vorhanden. Außerdem könnten die Auszeichnungen zu einer Art Elite führen. Einer Gruppe von Personen, die alle schon lange bei der Firma arbeiten oder die alle schon einmal für besondere Verdienste ausgezeichnet wurde. Dies könnte man Core Drive 1: Epic Meaning \& Calling zuordnen. Da die Maßnahme von fast jedem Core Drive zumindest ein wenig hat, kann man auf eine gut ausbalancierte Maßnahme schließen, die sowohl die intrinsische wie auch die extrinsische Motivation anspricht. Durch die starke Dominanz von Core Drive 2: Development \& Accomplishment steht die extrinsische Belohnung jedoch im Vordergrund. Trotz des Vorhandenseins von Core Drive 6: Scarcity \& Impatience sowie Core Drive 7: Unpredictability \& Curiosity ist die Maßnahme vor allem eine White-Hat-Maßnahme und eignet sich deswegen besonders gut, um eine Langzeitmotivation aufrechtzuerhalten.

In den Interviews wurde die Analyse teilweise bestätigt. Es wurde deutlich, dass bei dieser Maßnahme der Core Drive 2: Development \& Accomplishment durch die Mitarbeitenden als einer der Haupttreiber erkannt wird. Sie fühlen sich gut und motiviert dadurch, eine Auszeichnung zu erhalten. Dass nicht nur Firmentreue, sondern auch spezielle 
Anstrengungen belohnt werden, verstärkt dieses Gefühl noch. Das Gefühl, etwas erreicht $\mathrm{zu}$ haben und dafür wertgeschätzt $\mathrm{zu}$ werden, ist deutlich vorhanden. Auch Core Drive 5: Social Influence \& Relatedness ist deutlich vorhanden. Durch das Spektakel, das beim Verleihen veranstaltet wird, entsteht das Gefühl, dass die anderen die eigene Anstrengung honorieren. Außerdem freuen sich die Kollegen mit einem über den Erhalt, was der Sache einen deutlichen sozialen Bezug gibt. Dadurch, dass man eine Ehrenmedaille direkt nach dem sehr erfolgreichen Abschluss einer Arbeit erhält und nicht erst am Ende des Jahres beim Mitarbeitergespräch, wird der Aspekt des unmittelbaren Feedbacks von Core Drive 3: Empowerment of Creativity \& Feedback betont. Dieser Core Drive wird durch die Mitarbeitenden vermutlich stärker wahrgenommen, als dies bei der Planung angedacht war. Core Drive 6: Scarcity \& Impatience wird dagegen überhaupt nicht als solcher wahrgenommen. Zu Core Drive 1: Epic Meaning \& Calling sowie Core Drive 7: Unpredictability \& Curiosity fehlen in Bezug auf diese Maßnahme gezielte Aussagen in den Interviews. Auch hier muss deshalb davon ausgegangen werden, dass diese von den Mitarbeitenden kaum als solche wahrgenommen werden.

Die Verteilung der „Ämtlikarten“ ist in erster Linie ein Glücksspiel. Damit ist der vorrangige Core Drive dieser Maßnahme Core Drive 7: Unpredictability \& Curiosity. Dieser zielt darauf ab, dass man nicht genau weiß, ob und welche Aufgabe man erhält. Durch die Ziehung und die Kontrolle im Plenum ist auch Core Drive 5: Social Influence \& Relatedness vertreten. Aus Sicht der Gamification geht es hier vor allem darum, damit angeben zu können, dass man keine Aufgabe gezogen hat. Aber auch um das Teilen von Emotionen, sei dies Schadenfreude oder Mitgefühl. Etwas weniger stark, aber dennoch vorhanden, ist Core Drive 3: Empowerment of Creativity \& Feedback. Hier ist der Feedbackteil vertreten, indem man einerseits unmittelbar nach der Ziehung der Karten ein Feedback erhält, ob man ein Gewinner oder Verlierer ist, und andererseits am Ende der Woche das Feedback, ob die Aufgabe erfüllt wurde oder nicht. Dies geht ebenfalls ein bisschen in Richtung Core Drive 2: Development \& Accomplishment, wenn man am Ende der Woche verkünden kann, die Aufgabe erfüllt zu haben. Des Weiteren kann in der Maßnahme „Ämtlikarten“ etwas von Core Drive 1: Epic Meaning \& Calling gefunden werden. Dies da die Erledigung der Arbeit das Gefühl wecken kann, dass man die Aufgabe zum Wohle aller oder zum Wohle der Firma erledigt. In ganz kleinen Teilen vertreten ist außerdem Core Drive 4: Ownership \& Possession, da man die gezogene Aufgabe als seine eigene ansehen kann und deshalb versucht, diese bestmöglich zu erledigen, sowie auch Core Drive 6: Scarcity \& Impatience, da man nicht jederzeit erneut das Glück herausfordern und neue Karten ziehen kann. Die Maßnahme „Ämtlikarten“ ist klar right-brain-lastig und spricht damit die intrinsische Motivation an. Dies ist interessant, da es eigentlich um die Verteilung unliebsamer Arbeiten geht. Mit dem starken Fokus auf Core Drive 7: Unpredictability \& Curiosity ist es zudem eher eine Black-Hat-Gamification-Maßnahme, was darauf schließen ließe, dass die Motivation lediglich kurzfristig ist und nach einer gewissen Zeit nachlässt. Die Maßnahme hat kumuliert jedoch genügend kleine White-Hat-Elemente, dass auch eine gewisse Langzeitmotivation vorhanden sein dürfte. 

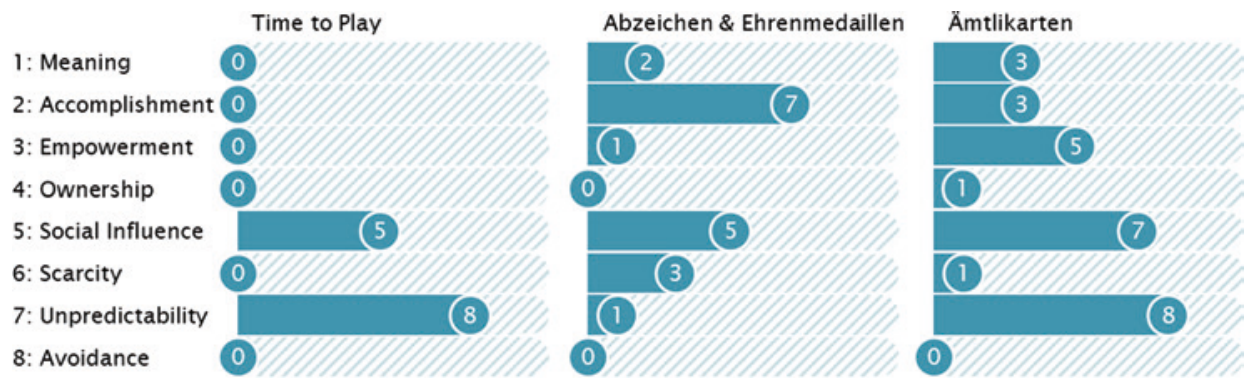

Abb. 4.4 Auswertung der Maßnahmen nach dem Octalysis-Modell (Eigene Darstellung)

Durch die Mitarbeitenden wird der Core Drive 7: Unpredictability \& Curiosity in dieser Maßnahme sofort erkannt. Dem zufälligen Verteilen der Karten wird Gamblingcharakter nachgesagt und es ist auch bei der Wahrnehmung das vorherrschende Gefühl. Auch Core Drive 5: Social Influence \& Relatedness wird klar erkannt und durch die Maßnahme gestärkt. Die Ziehung wird als gesellschaftliches Ritual angesehen und verbindet dadurch, dass man gegenseitig mitfühlt oder gemeinschaftliche Schadenfreude zum Ausdruck bringt. Auch in der Gleichstellung sämtlicher Mitarbeitenden des Unternehmens wird ein starker Aspekt von Core Drive 5: Social Influence \& Relatedness gesehen. Core Drive 1: Epic Meaning \& Calling wird als solcher wahrgenommen, tendenziell noch stärker als in der Planung der Maßnahme erwartet. Das Gefühl etwas Unliebsames zum Wohle aller zu erledigen, ist definitiv vorhanden. Auch Core Drive 2: Development \& Accomplishment sowie Core Drive 3: Empowerment of Creativity \& Feedback werden mehr oder weniger so wahrgenommen, wie dies für diese Maßnahme vorgesehen war. Durch das Feedback in der darauffolgenden Woche ist man eher gewillt, die Aufgabe zu erledigen und man hat auch das Gefühl, etwas erreicht zu haben. Core Drive 4: Ownership \& Possession und Core Drive 6: Scarcity \& Impatience werden hingegen gar nicht als solche wahrgenommen. Keine Aussage lässt darauf schließen, dass man die Aufgabe zuverlässiger erledigt, da man diese als seine eigene ansieht. Auch hat offensichtlich niemand das Verlangen, das Glück erneut herauszufordern, da alles, was man bekommen kann, als eher negativ angesehen wird.

Nachfolgend wurden die mit den Mitarbeitenden der Firma Acme Apps AG geführten Interviews weiter ausgewertet. Das Ziel der Interviews war es zu untersuchen, wie gut die drei vorgestellten Maßnahmen aufgenommen werden, ob diese zur Unternehmenskultur beitragen und ob dadurch die Arbeitsmotivation gesteigert werden kann. In einem ersten Schritt werden dafür die empirisch erhobenen Daten vorgestellt, die anschließend in einem zweiten Schritt interpretiert wurden (Abb. 4.4). Insgesamt wurden Interviews mit vier Mitarbeitenden geführt. Da die Option der Anonymisierung von einzelnen wahrgenommen wurde, werden die Aussagen in der Arbeit keinen spezifischen Urhebern zugewiesen. 
In Bezug auf die Unternehmenskultur sind sich die Interviewpartner einig, dass diese bei Acme Apps AG sehr offen sei. Auch das demokratische, selbstbestimmende, beziehungsweise das Mitspracherecht aller, wird als besonders großer Pluspunkt angesehen. Die Bezeichnung „familiär“ wird außerdem mehrmals als Charakterisierung genannt. Dies entspricht der durch die Führung angestrebten offenen Kultur auf Augenhöhe, die durch ein Mitspracherecht aller getragen wird. Der Einfluss der Unternehmenskultur auf die Arbeitsmotivation wurde von allen Interviewpartnern als sehr stark beurteilt. Besonders motivierend wirke sich dabei das Mitspracherecht aus, wie auch das Gefühl, Teil eines Teams, eines größeren Ganzen zu sein. Aber auch die Arbeitsgestaltung, beziehungsweise die Work-Life-Balance sowie die Arbeit selbst wurden genannt. Insofern kann gefolgert werden, dass die Mitarbeitenden durch die vorherrschende Kultur im Unternehmen motiviert werden, die zu erledigende Arbeit selbst aber auch motiviert.

Die Maßnahme „Time to Play“ kommt bei allen Befragten gut bis sehr gut an. Viele sehen den Wert der Pause darin, dass man anschließend wieder motivierter und konzentrierter arbeiten könne. Wann genau der Alarm losgeht, wird dabei kaum als Ungewissheit bewertet. Zwar wird es als toll angesehen, dass nicht alles streng geregelt und an fixe Zeiten gebunden ist, doch scheint dies mittlerweile beinahe zum Tagesablauf zu gehören und wird nicht mehr als etwas Spezielles wahrgenommen. Als sehr positiv wird die soziale Komponente wahrgenommen. Nicht nur bringe der Alarm auch Leute zum Spielen, die sonst eher weniger mitmachen, sondern lasse beim Spielen auch persönliche Kontakte und Gespräche zu, die sich nicht um die tägliche Arbeit drehen. Man lerne sich so auf eine andere Art kennen als während der ,,produktiven“ Arbeit.

Die Maßnahme „Abzeichen \& Ehrenmedaillen“ wird grundsätzlich als etwas sehr Positives angesehen. Die Mitarbeitenden finden es schön, wenn sie für gute Arbeit ein besonderes Lob erhalten. Dass Firmentreue belohnt wird, wird dabei als gerecht betrachtet. Insbesondere in einer Zeit, in der es nicht mehr selbstverständlich ist, dass man allzu lange beim gleichen Arbeitgeber bleibt. Der Erhalt einer Ehrenmedaille wirke sich durchaus motivierend aus und bestärke einem in der Arbeit, die man tue. Auch motiviere, dass ein geleisteter besonderer Aufwand anerkannt und honoriert werde.

Wenn jemand anderes eine Medaille erhält, wird dies dabei nicht als negativ betrachtet, sondern man freut sich mit diesen zusammen. Dass die Medaillen fair verteilt werden, scheint aber dennoch ein wichtiger Aspekt zu sein. Da die Medaille jedoch aus unterschiedlichsten Gründen vergeben werden kann, erscheint dies bisher nicht als Problem. Mehrmals wird erwähnt, dass diese Maßnahme die Motivation insgesamt stärke. Besonders schön sei dabei, dass dies nicht nur pro forma gemacht werde, sondern wirklich ein Spektakel sei. Es wird aber auch erwähnt, dass die Maßnahme die tägliche Motivation zur Erledigung der Tagesarbeit kaum beeinflusse.

Die Ziehung der „Ämtlikarten“ wird als wöchentliches Highlight angesehen, das eine gute Stimmung verbreite. Besonders gut wird dabei die Tatsache aufgenommen, dass sich alle an der Ziehung beteiligen. Nicht nur verleihe dies dem Ritual eine soziale Komponente, sondern es wird darin trotz der Zufallskomponente auch die Fairness gesehen. 
Jedes „Ämtli“ könne jeden treffen. Dass dies dabei womöglich zweimal hintereinander dieselbe Person sein kann, wird nicht als unfair betrachtet, sondern als Pech. Die Maßnahme wird somit auch als spannend angesehen und es wird ihr gar Glücksspielcharakter nachgesagt. Die Mehrheit der Befragten ist sich einig, dass die Maßnahme die Kultur im Unternehmen positiv beeinflusse. Sie fördere die Gleichstellung aller Mitarbeitenden, was ein Kulturziel der Acme Apps $A G$ darstellt. Zudem profitiere auch das Gemeinschaftsgefühl davon. Dennoch wird auch einmal erwähnt, dass die Maßnahme keinen Einfluss auf die Motivation für die restlichen Arbeiten habe.

Die vorherrschende Kultur bei Acme Apps $A G$ wird von den Interviewpartnern als einer der zentralen Erfolgsfaktoren der Firma angesehen. Dabei wird es als erfrischend betrachtet, dass das Unternehmen hier eine etwas andere Herangehensweise hat als andere Unternehmen. Die analysierten Maßnahmen helfen dabei, die Kultur und speziell den Teamzusammenhalt zu fördern. Es wird aber auch angemerkt, dass die Maßnahmen allein keine gute Kultur ausmachen, sondern lediglich eine existierende fördere. Denn auch andere Unternehmen haben Maßnahmen für ähnliche Szenarien, haben diese lediglich anders aufgebaut. Es ist also nicht nur die Vergabe kleiner Aufgaben oder das Honorieren von Unternehmenstreue, die motiviert, sondern vor allem, wie dies in einem vorhandenen kulturellen Kontext umgesetzt wird.

\subsection{Diskussion und Schlussfolgerungen}

Die vorliegende Studie soll Aufschluss darüber geben, ob und welche Gamification-Elemente in den Maßnahmen von Acme Apps AG integriert wurden. Anhand der Auswertung der Maßnahmen kann das Vorhandensein von Gamification klar bestätigt werden. Alle drei Maßnahmen beinhalten Elemente aus mehreren Core Drives gemäß dem Octalysis-Modell. Relativiert wird dieses Ergebnis durch die Prämisse des Modells, dass ohne Anwesenheit mindestens eines Core Drives überhaupt keine Aktion stattfindet und dass auch nicht spezifische Maßnahmen, die nicht nach Gamification-Kriterien gestaltet wurden, einen geringen Octalysis-Score aufweisen. Es kann deshalb geschlussfolgert werden, dass zumindest die Maßnahme „Time to Play“ nicht als spezifische Gamification-Maßnahme aufgefasst werden sollte. Abschließend sollten die Maßnahmen als Teile von etwas größerem Ganzen angesehen werden. So trägt jede Maßnahme zum Ziel bei, die Unternehmenskultur bei Acme Apps AG zu beeinflussen, ohne dass diese notwendigerweise als gamifiziert betrachtet werden.

Die zweite Zielsetzung war es, zu eruieren, ob die Maßnahmen von Acme Apps AG die Unternehmenskultur tatsächlich beeinflussen. Diese Fragestellung kann anhand der ausgewerteten Interviews ebenfalls positiv beantwortet werden. Von allen Interviewpartnern wird mehrmals erwähnt, dass die Maßnahmen insbesondere das Zusammengehörigkeitsgefühl unter den Mitarbeitenden fördern. Sie bauen einen Teamspirit auf und geben den Mitarbeitenden das Gefühl ein Teil von Acme Apps AG zu sein. Damit tragen die Maßnahmen klar zur angestrebten offenen Kultur auf Augenhöhe bei. 
Die dritte Zielsetzung war es, zu erörtern, ob die Unternehmenskultur von Acme Apps $A G$ die Motivation der Mitarbeitenden dahingehend anregt, sich für ihre Arbeit einzusetzen.

Auch diese Fragestellung kann aufgrund der Antworten der Interviews mit „Ja“ beantwortet werden. Die Interviewpartner haben übereinstimmende Aussagen gemacht, dass die Unternehmenskultur bei Acme Apps AG ein wichtiger Motivator für sie ist. Spezifisch wurde hier die gefühlte Mitverantwortung für das Team, beziehungsweise das Unternehmen erwähnt, das sich aufgrund der Kultur ergibt. Dies bewirkt, dass die Mitarbeitenden einen tieferen Sinn in ihrer Tätigkeit sehen und dadurch auch längerfristig motiviert bleiben.

Die Studie zeigt, dass Gamification ein angemessenes Werkzeug für Kulturveränderung sein kann. Durch die Anwendung verschiedener Core Drives können vielfältige Maßnahmen gezielt gestaltet und auf ihre Wirkungen getestet werden. Gleichzeitig wird deutlich, dass nicht jede Maßnahme unbedingt gamifiziert werden muss, sondern es eine Mischung aus Maßnahmen mit unterschiedlich stark ausgeprägten Gamification-Elementen geben kann. Der Einsatz von Gamification wurde von den Betroffenen und Beteiligten als insgesamt positiv wahrgenommen. Für Führungskräfte eröffnet sich hier ein Feld an Möglichkeiten, dass die digitale Transformation erst ermöglicht hat, sowohl von der technischen Seite als auch, was die kulturelle Akzeptanz und Offenheit von Mitarbeitenden anbelangt. Insgesamt ist Gamification noch ein junges und wenig ausgelotetes Gebiet, bei dem es sich lohnt, selbst einfache, kleine Experimente durchzuführen.

\section{Literatur}

Adams, E. W. (2014). Fundamentals of game design (3. Aufl.). London: Pearson Education Inc.

Bartle, R. (1996). Hearts, clubs, diamonds, spades: Players who suit MUDs. Colchester: MUSE Ltd. http://mud.co.uk/richard/hcds.htm. Zugegriffen: 8. Apr. 2018.

Burke, B. (2013). The gamification of business. Forbes, 21.01.2013. https://www.forbes.com/sites/ gartnergroup/2013/01/21/the-gamification-of-business. Zugegriffen: 12. Apr. 2018.

Chou, Y.-K. (2012). http://yukaichou.com/gamification-examples/octalysis-complete-gamification-framework/\#.WvX8pIiFO70. Zugegriffen: 8. Apr. 2018.

Chou, Y.-K. (2016). Actionable gamification. Freemont: Octalysis Media.

Chou, Y.-K. (2018). http://www.yukaichou.com. Zugegriffen: 8. Apr. 2018.

Coonradt, C. (2012). The game of work (revised and updated Aufl.). Layton: Gibbs Smith.

Dale, S. (2014). Gamification: Making work fun, or making fun work? Business Information Review 31(2), 82-90. http://journals.sagepub.com/toc/bira/31/2/82. Zugegriffen: 10. Apr. 2018.

De Luca, A. (2017). What is salary? Bachelor Thesis. Bern: Berner Fachhochschule.

Deterding, S., et al. (2011). Gamification: Toward a definition. Vancouver: o. V. http://hci.usask.ca/ uploads/219-02-Deterding,-Khaled,-Nacke,-Dixon.pdf. Zugegriffen: 10. Apr. 2018.

Engelhard, M. (2014). Gamification in Theorie und Praxis. Masterarbeit. Würzburg: Julius -Maximilians-Universität, Lehrstuhl für Wirtschaftsinformatik und Systementwicklung.

Herger, J., \& Strobl, H. (2018). Unternehmenskultur in der Praxis. Wiesbaden: Springer. 
Jost, H. R. (2003). Unternehmenskultur. Zürich: Orell Füssli.

Kapp, K. M. (2012). The gamification of learning and instructions. San Francisco: Pfeiffer.

Kotter, J. P., \& Heskett, J. L. (1992). Corporate culture and performance. New York: Free Press.

Levy, H. P. (2015). 5 key trends in Gartner's 2015 digital marketing hype cycle. Gartner, 14.10.2015. https://www.gartner.com/smarterwithgartner/five-key-trends-in-gartners-2015-digital-marketing-hype-cycle/. Zugegriffen: 10. Apr. 2018.

Maier, G. W., \& Kirchgeorg, M. (2018). Motivation. Gabler Wirtschaftslexikon. https://wirtschaftslexikon.gabler.de/definition/motivation-38456. Zugegriffen: 20. Apr. 2018.

McDonald, E. (2017). The global games market will reach $\$ 108.9$ Billion in 2017 with mobile taking 42\%. Newzoo, 20.04.2017. https://newzoo.com/insights/articles/the-global-games-market-will-reach-108-9-billion-in-2017-with-mobile-taking-42/. Zugegriffen: 12. Apr. 2018.

McGonigal, J. (2012). Reality is broken. London: Vintage.

Mühlenhof, M. C. (2018). Chefsache intrinsische motivation. Wiesbaden: Springer.

Newman, D. (2016). Top 10 trends for digital transformation in 2017. Forbes, 30.08.2016. https://www.forbes.com/sites/danielnewman/2016/08/30/top-10-trends-for-digital-transformation-in-2017/. Zugegriffen: 12. Apr. 2018.

Nicholson, S. (2012). A user-centered theoretical framework for meaningful gamification, Madison. http://scottnicholson.com/pubs/meaningfulframework.pdf. Zugegriffen: 14. Apr. 2018.

Norman, D. A. (2013). The design of everyday things (Revised and expanded Aufl.). New York: Basic Books.

Pink, D. H. (2011). Drive: The surprising truth about what motivates us. New York: Riverhead Books. (Reprint).

Ryan, M. et al. (2013). Why gamification is serious business. Outlook, 2013(1): Accenture. https:// www.accenture.com/us-en/insight-outlook-why-gamification-is-serious-business. Zugegriffen: 14. Apr. 2018.

Sackmann, S. (2002). Unternehmenskultur. München: Hermann Luchterhand.

Schein, E. H. (1984). Coming to a new awareness of organizational culture. Sloan Management Review, 25(2), 3-16. http://www.sietmanagement.fr/wp-content/uploads/2016/04/culture_ schein.pdf. Zugegriffen: 14. Apr. 2018.

Stieglitz, S. (2015). Gamification - Vorgehen und Anwendung. Praxis der Wirtschaftsinformatik, 52(6), 816-825.

Wikipedia. (2018). Generation Y. https://de.wikipedia.org/wiki/Generation_Y. Zugegriffen: 14. Apr. 2018.

Wiktionary. (2017). Gamification. https://en.wiktionary.org/wiki/gamification. Zugegriffen: 14. Apr. 2018.

Wiktionary. (2018). -ification. https://en.wiktionary.org/wiki/-ification. Zugegriffen: 14. Apr. 2018.

Zelesniack, E., \& Grolman, F. (2018). Unternehmenskultur. https://organisationsberatung.net/ unternehmenskultur-kulturwandel-in-unternehmen-organisationen/. Zugegriffen: 20. Apr. 2018.

Ellenberger, Thomas (B.Sc. BA/thomas.ellenberger@gmx.ch) Abgeschlossenes, berufsbegleitendes Bachelorstudium in Betriebsökonomie mit Vertiefung in Management in Practice: Strategy. Während dem Studium angestellt als Implementation Manager bei der Swisscom (Schweiz) AG sowie als Sachbearbeiter Fachstelle Cicero beim Berufsbildungsverband der schweizerischen Versicherungswirtschaft (VBV) 
Harder, Deane (Prof. Dr./deane.harder@bfh.ch) Tätigkeit in Lehre und Forschung an der Berner Fachhochschule, Departement Wirtschaft. Schwerpunkte sind Innovation, Verhaltensökonomie und Systemik im Bereich Nachhaltigkeit. Vorher 10 Jahre Praxiserfahrung in der Unternehmensberatung und -entwicklung. Studium und Promotion in Biologie an den Universitäten Freiburg i. Br. (Deutschland) und Otago University (Neuseeland).

Brechbühler Pešková, Marie (Prof. Dr./marie.brechbuehler@bfh.ch) ist in der Lehre und Forschung an der Berner Fachhochschule, Departement Wirtschaft tätig. Sie unterrichtet insbesondere Module in den Bereichen „Nachhaltige Wirtschaft, Strategie und Innovation und International Business. Sie leitet der Forschungsschwerpunkt „Nachhaltigkeit“ und widmet sich in Ihre angewandte und praxisorientierte Forschungstätigkeit der Themen „Transformation zur nachhaltigen Gesellschaft und Wirtschaft“, „Nachhaltige Kreisläufe: Sustainable Consumption, Circular Economy, nachhaltige Geschäftsmodelle, Recycling“ sowie „Corporate Sustainability / CSR/ social Entrepreneurship“.

Open Access Dieses Kapitel wird unter der Creative Commons Namensnennung 4.0 International Lizenz (http://creativecommons.org/licenses/by/4.0/deed.de) veröffentlicht, welche die Nutzung, Vervielfältigung, Bearbeitung, Verbreitung und Wiedergabe in jeglichem Medium und Format erlaubt, sofern Sie den/die ursprünglichen Autor(en) und die Quelle ordnungsgemäß nennen, einen Link zur Creative Commons Lizenz beifügen und angeben, ob Änderungen vorgenommen wurden.

Die in diesem Kapitel enthaltenen Bilder und sonstiges Drittmaterial unterliegen ebenfalls der genannten Creative Commons Lizenz, sofern sich aus der Abbildungslegende nichts anderes ergibt. Sofern das betreffende Material nicht unter der genannten Creative Commons Lizenz steht und die betreffende Handlung nicht nach gesetzlichen Vorschriften erlaubt ist, ist für die oben aufgeführten Weiterverwendungen des Materials die Einwilligung des jeweiligen Rechteinhabers einzuholen.

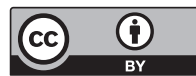

